\title{
Potential of Geospatial Mashups in Promoting Tourism Resources in South East Asia: A Case Study
}

\author{
Somnath Chaudhuri ${ }^{1}$ and Panacea Yamin ${ }^{2}$
}

\begin{abstract}
Tourism has emerged as a new form of service industry for alternative and sustainable socio-economic development of a country. Geographic Information System (GIS) is gradually becoming an integral part of this service industry. In spite of having diverse culture, environment and heritage, a number of Southeast Asian tourism sites are still not integrated into the global tourism map. Although these unexplored locations are in the process of emerging as popular global tourist destinations, there is lack of available online information. The current research work has been conducted in one of the Southeast Asian nations to analyze the existing geospatial information and the tourist inflow of that particular region. This paper further discusses the basic architecture and the capabilities of Geospatial Mashups in identifying potential tourism resources and assisting in effective decision making. The final section of the study proposes a model for Public Participation Geoportal and its strategic implementation for effective tourism management and promotion not only in the study region but also in other countries in Southeast Asia. QGIS 2.18.12 software has been used to analyze existing tourism data and to design the proposed model.
\end{abstract}

Keywords - GIS, Geospatial, MGIST, OGC, Web 2.0

\section{INTRODUCTION}

In the face of twenty-first century, tourism is one of the highest income generating industries over the globe. The effective management and promotion of this smokeless service-industry can help in significant economic development of a nation. According to World Travel and Tourism Council (WTTC) 2017 report on the global economic impact, in the year 2016, the direct contribution of Travel \& Tourism to global GDP was USD 2,306 billion i.e., $3.1 \%$ of the total GDP, and is forecast to rise by $3.8 \%$ in 2017 , and to rise by $4 \%$ per annum, from 2017-2027, to USD 3,537.1 billion i.e., 3.5\% of total GDP in 2027 [1]. The World Tourism Organization (UNWTO) in the recent issue of the World Tourism Barometer mentioned that, "International tourist arrivals worldwide grew by 6\% in January-April 2017 compared to the same period last year". The more tourism is becoming popular; the travelling pattern among the tourists is also changing dynamically. The trend has now shifted

Somnath Chaudhuri ${ }^{1}$ is with the Faculty of Engineering, Science and Technology, Maldives National University, Republic of Maldives

Panacea Yamin ${ }^{2}$, is a freelance researcher in digital marketing in Myanmar towards adventure tourism having scope of intercultural exchanges. New adventures, unique culture and heritage are profound in the Southeast Asian region especially, in the developing countries like: Malaysia, Cambodia, Indonesia, Philippines, Laos, Thailand, Vietnam and Myanmar. Natural resources including sea beaches, rivers, forests, mountains and lakes, valuable heritage, rich historical legacy, and traditional culture; are the hordes of treasure helping in developing tourism in the Southeast Asian countries. International tourist arrival in this region is increasing substantially. Figure 1 (below) depicts the statistics of international tourist arrivals in this region from 2010 to 2016.

Number of Tourists (in million)

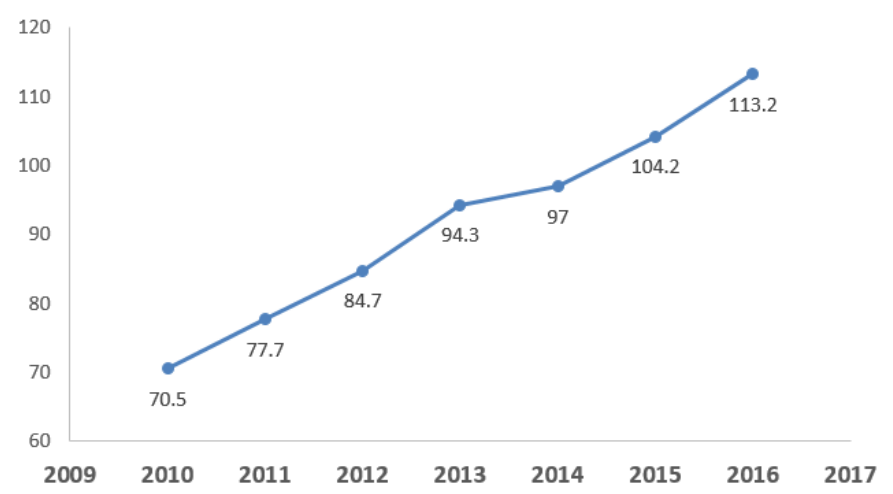

Source: World Tourism Organization (UNWTO, 2017)

Fig. 1 International tourist arrivals in Southeast Asia region

Republic of the Union of Myanmar is one of the developing nations in Southeast Asia. It has an area of 676,577 square $\mathrm{km}$, extending $1,931 \mathrm{~km}$ in the North-South and $925 \mathrm{~km}$ in the East-West.

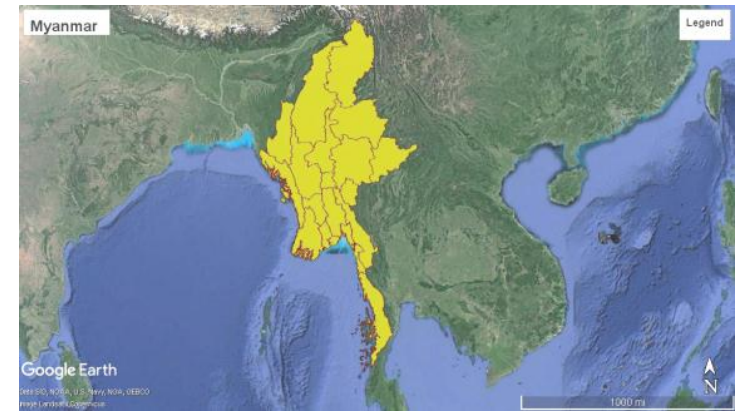

Source: Designed using Google Earth Pro'2017

Fig. 2 Server-side Mashups Architecture 
Myanmar has a wide range of natural diversity; the snowcapped mountain in Putao, Inle Lake in the Shan state, Ngapali beach located in Rakhine state, Mergui Archipelago consists of 800 islands located in the southern part of Myanmar. Beside these, the country has the charming capital city of Yangon, the temple city of Bagan, the cave housing in Pindaya, the Golden Rock in Mon state and many more [2]. All these charming realities are beckoning tourists from far and wide to this beautiful Southeast Asian country. According to the Ministry of Hotel and Tourism (2015), Myanmar it is a land of astounding beauty and charm that moved the famous English author Rudyard Kipling to pen these apt words: "quite unlike any land you will ever know" [3]. Since 2010, in line with the reform strategies and economic liberalization of Myanmar, the Ministry of Hotels and Tourism (MoHT) has been placing considerable emphasis on developing and managing tourism in sustainable and responsible manner [4]. In a report by the World Travel and Tourism Council (WTTC) on the Economic Impact 2017 in Myanmar it was depicted that, the direct contribution of Travel \& Tourism to GDP was 2577.6 billion Burmese Kyat (MMK) i.e., 3\% of total GDP in 2016, and is forecast to rise by $3.5 \%$ in 2017, and to rise by $7.4 \%$ per annum, from 2017-2027, to 5,425.5 billion MMK i.e., 3.2\% of total GDP in 2027 [1]. Recently, in another report by WTTC, in the year 2016 Myanmar stands 87 th out of 185 countries in terms of relative importance of Travel \& Tourism's total contribution to GDP [1]. MoHT in the Myanmar Tourism Master Plan (2013-2020) have categorized the tourism destinations with their unique attraction features.

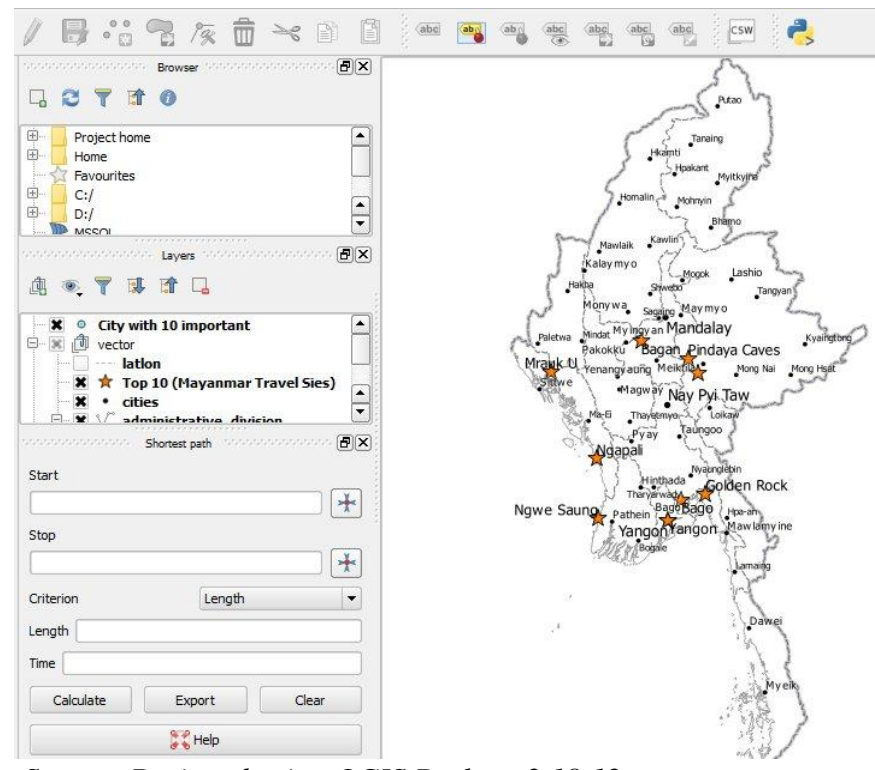

Source: Designed using QGIS Desktop 2.18.12

Fig. 3 Map of Myanmar:Important cities and top ten tourist locations

Top ten popular tourist destinations of Myanmar (Inle lake, Shwedagon Pagoda, Golden Rock, Bagan, Bago, Yangon, Ngapali, Mrauk U, Pindaya Caves and Ngwe Saung) along with nearly 50 important cities are depicted in the map (figure 3). The map has been designed using open source cross-platform GIS software, Quantum GIS (QGIS). This software helps in integrating and analysis of heterogeneous geo-referenced data.

\section{Geospatial MashuPS}

Information and Communication Technology (ICT) can lead tourism to emerge as a new mantra for alternative economic development [5]. During the mid-1970s, Harvard Laboratory Computer Graphics developed the first ever vector GIS, ODYSSEY GIS. This development revolutionized the GIS software development process and facilitates the increase in geo information systems in the next generation.

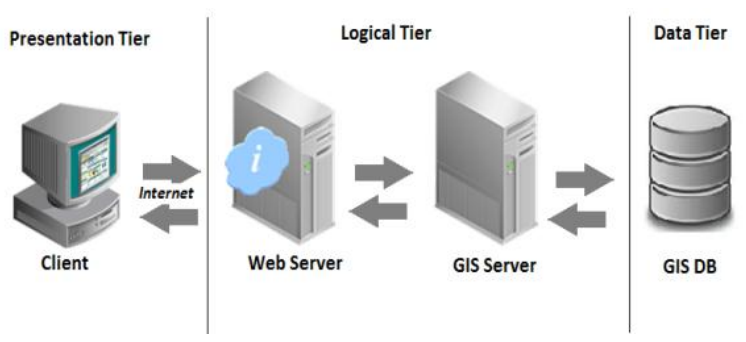

Fig. 4 Web GIS Workflow

The broad Web GIS workflow is depicted in the figure 4 (above). The workflow has been categorized into three sections, namely: Presentation tier, Logical tier, and Data tier. The query execution and the data processing and interpretation are performed in the logical tier. Depending upon the nature of the query the final output can be a simple map or, a Mashup map, or, can even be a tabular data set.

The three tier Web GIS system is definitely an easier and user-friendly application to get access to geospatial data over the internet just on a click. The quality of the data in the backend GIS database, decides the standard of the result generated by the Web GIS application. If the database is not periodically updated the search result can face data update anomaly. At the same time, it is not feasible to maintain and update the overall database by a single organizational unit. Slowly, it was becoming obvious that a collaborative contribution is required to maintain the quality and an up-todate Web GIS database. This concept of collaborative contribution for the Geospatial system led to the new avenue of technology called Geospatial Mashups. A Mashup is a web page that dynamically integrates contents from multiple web sources. Mashup having at least one georeferenced component is called a Geomashup. It is the modern technology of providing web mapping or GIS services globally. Integration of multiple data layers or datasets, often from multiple sources, is one of the most common functional requirements of GIS applications [6]. Recently, a new term has been coined in the Web Community: "Web 2.0". The primary aspect of this second generation online community is its collaborative and interactive feature. Today any nontechnical web user can also create or update content on the web. According to OGC (2002), integration of multiple data layers from multiple sources, is one of the most common and 
effective functional requirements of Web GIS applications [7]. In context to Web GIS, a Mashup is the process of merging multiple sources of data, both spatial and nonspatial, into a single integrated spatial display. It is about extracting spatial data from a non-spatial source and combining with other spatial data and finally displaying it on a map [8]. But just embedding a video or an image on a web page is not a Mashup. The factual Mashup technology allows the contributor to host, update, customize and maintain global data without having any constraints. So that even heterogeneous data from varied sources will be combined in a common platform, accessible by any internet users just on a click. The basic architecture of Web GIS consists of two application layers, server end and client or browser end. The Mashup operation can be applied in both the ends. When the Mashup operation is executed on the server end, it is called a Server-side Mashup and when it is on the client end it is called as Browser-side Mashup. The mode of operation for both client-side and browser-side Mashup is same. In both the cases, the Web browser sends requests to different services, receives the responses and displays the desired composite results. The advantage of server-side Mashups over the clientside is that the server has the capability of using more powerful hardware and software than the browser. These can generate a better output result. But these Mashups also require specialized programming tools and complex-server side programming, which includes more technical skills during development and deployment. Because of this complex programming, the original Server-side Mashups are largely limited to professional programmers. Client-side Mashups do not use particular server that acts as proxy, but combine different data and components with the client browser. Most of the today's Mashups are Browser or Client side. They basically use lightweight programming; as a result it has become easy to develop and maintain. The common georeferenced Mashup design consists of three components: Basemaps, Operational Layers and Tools.

- Base-maps provide the initial framework for any Geomashup. It is the frame of reference.

- Operational Layers known as the thematic layers, usually embedded on the top of a base maps.

- Tools: It can execute logical and analytical functions.

\section{IMPLICATIONS OF GEOSPATIAL MASHUPS IN TOURISM}

GIS is an information system that provides functions including visual presentation about the tourism destination, advanced analyses, etc., of digital geospatial information by processing it in an integrated manner on electronic maps of the tourist site [9]. Mcadam (1999) discussed the scope and impact of GIS in tourism planning and highlighted that, GIS add value to decision making by the strategic application of data analysis, modeling and forecasting [10]. In the year 2004, Turk and Gumusay conducted a research work in the Eminonu district, Turkey using GIS design and network analysis depicting the GIS possibilities for destination management [11]. Zaragoza is one of the cultural heritage centers and the fifth most populated city of Spain. The city council of Zaragoza has deployed a tourist promotional Mashup, named eZaragoza. It provides only tourism related information for both internal and external entities [12]. Gradually, the trend of GIS was shifting towards Web GIS and finally with advent of Web 2.0, tourism information got influenced by Georeferenced Mashups. Anderson and Souleyrette (2002) suggested a dynamic modeling technique to assess tourist information technology implementation by integrating a regional travel demand model and microsimulation application package in a Geospatial environment [13]. Feick and Hall (2000) highlighted on the application of Geomashup technology within a customized software design to facilitate multiple participants from different regions of West Indies to contribute in a common tourism database [14]. Berger et al. (2007) illustrated an e-Tourism environment based on community-driven approach to create a lively society of travelers who can share travel experiences and recommend tourist destinations [15]. Singh et al. (2011) suggested a georeferenced information system (GeoRIST) to customize potential tourist's individual needs and preferences and relevant information will be available while being on the move [16]. Geospatial Mashups are contributing in the overall tourism development and management by providing Exploratory Spatial Analysis (ESA), Value-added Marketing and Geotagged Mashup ads. Thus, it is helping in strategic management and promotion of potential tourist destinations in a collaborative environment. Geoportal of few countries have also incorporated the Mashup technology to keep it dynamic and interactive with most up to date information. One such example is the Luxembourg National Geoportal as depicted in Figure 5 (below).

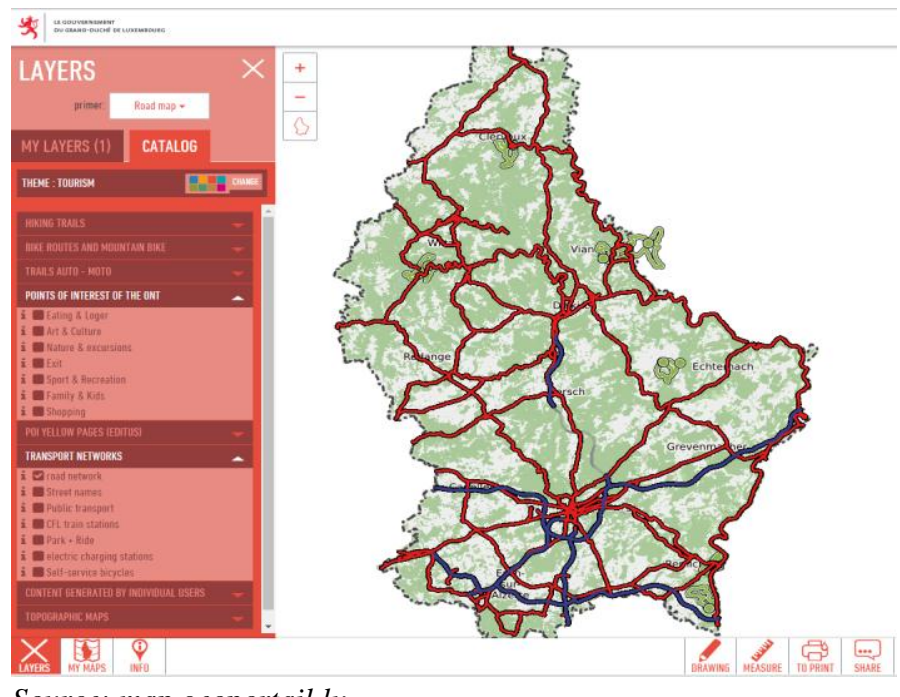

Source: map.geoportail.lu

Fig. 5 Geoportal Example (Luxembourg-Theme Tourism)

Any potential tourist can access this national geoportal. It has categories like, Bike Routes, Hiking trails, Points of Interest, Transport Networks, Topographic Maps etc. designed mainly for the tourism theme. To add value each of the categories are classified into subcategories like: Eating 
and Loger, Sports and Recreation, shopping etc. under the category Points of interest.

All these metadata repositories under each category provide detailed geospatial information just on click. It also provides Web 2.0 features in a Mashup environment. Any user can create his own map, a bike or hiking trail or can just be a café stroll. It can be readily updated in the portal using "Content Generated by Individual Users" and it will be included in the portals metadata repository for further reference by any potential tourist.

Another example is from Maldives. Eatolls.com (under process) provides Geospatial information in an interactive map interface. Figure 6 (below) depicts the query result of sea route information for a particular resort from the International airport of Maldives.

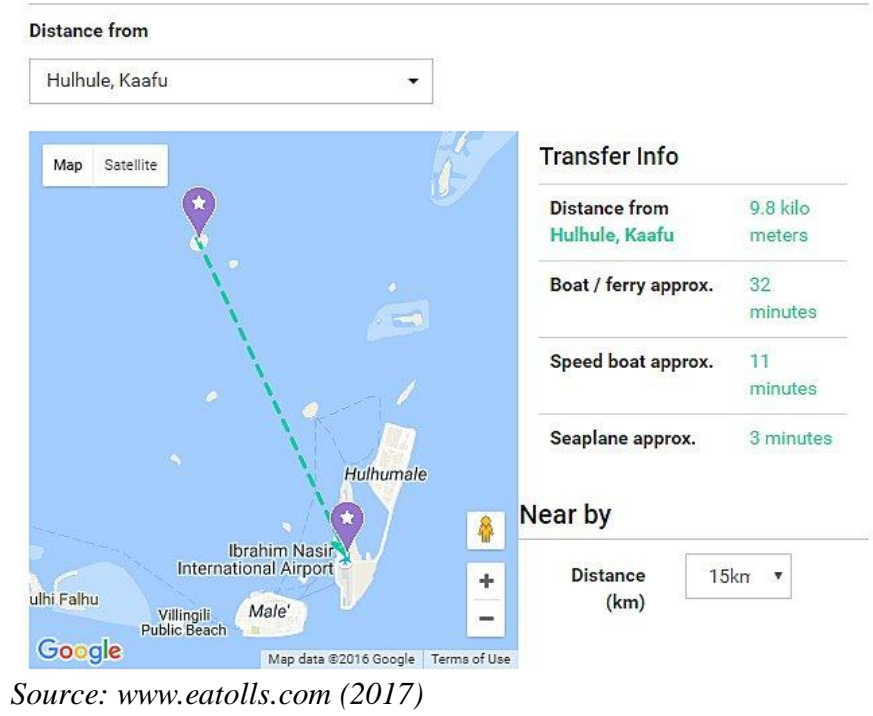

Fig. 6 Directional Information by Geo-tagged Website

Few other impressive national geoportals supporting tourism management having dynamic Mashup features are: Philippines (www.geoportal.gov.ph/apps/tourism)

Rwanda (http://rwandageoportal.rnra.rw)

Valencia (en.comunitatvalenciana.com/geoportal)

India (http://bhuvan.nrsc.gov.in/tourism)

\section{Myanmar Tourism: Online Available Resources}

Over the past 5 years tourist arrival in Myanmar has been increasing steadily. Figure 7 (below) supports the same. According to Myanmar Tourism Statistics, the total number of foreign tourists in the year 2014 was 11,31,624, it increased by $15 \%$ and in the 2015 it reached to $13,01,583$ [2]. The Ministry of Hotel and Tourism, the Republic of the Union of Myanmar maintains a user-friendly website dissipating detail tourism information. The multimedia and information enriched website has regular updates and has the set of metadata repository like, annual tourist inflow statistics, visitors guide book, e-brochures and e-pamphlets, it even highlights current news on Myanmar tourism. The website also provides helpful details about popular tourist locations with information on local festivals highlighting the areas where special permission is required and guidelines for the convenience of international visitors. The website provides georeferenced information about the MoHT branch offices, tourist police offices, information centers, and hotels [17]. In terms of static information MoHT website is helpful for potential tourists. On the other hand, to improve the capacity for analysis and decision making by a wide variety of stakeholders - including the United Nations, the Myanmar Information Management Unit (MIMU) provides effective information management services.

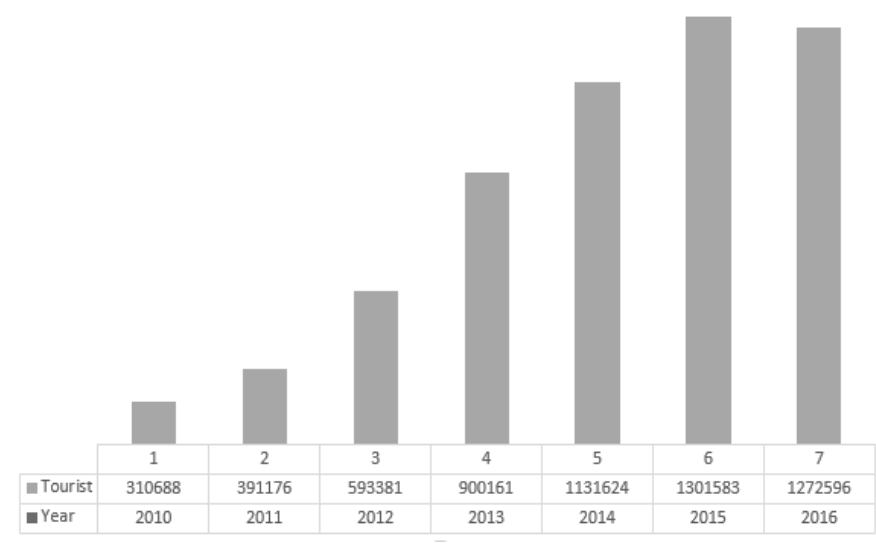

Source: Myanmar Tourism Statistics, 2017 [2]

Fig. 7 Tourist Arrival Trend in Myanmar

It provides information management services to reinforce analysis and decision-making for sustainable development in Myanmar. MIMU maintains a common data and information repository with spatial and non-spatial data from various sources on all sectors, countrywide. This information is then made widely accessible for different stakeholders in the form of georeferenced maps and databases. MIMU supports the coordination, planning and implementation of humanitarian and development activities [18]. The metadata repository contains huge set of georeferenced database, with customized filtering and search options. In terms of e-governance data for analysis and decision-making, MIMU website plays a vital role. But it lacks in precise tourism related information.

Few interactive mobile apps on Myanmar Tourism are available online. Among those, "Burma (Myanmar) - Free Travel Guide" is impressive. It provides detail information about the Essentials (Phrasebook, Eat, Drink, Sleep, Buy, Stay Safe, Stay Healthy, Respect, and Contact) for potential tourists along with General Information (Overview, History, Government \& politics, Economy, Demographics, Culture, Geography, and Education) and Transportation Facilities about Myanmar. But the most attractive and useful features of this mobile app are Attractions: Top 10, Maps, Regions, Top Cities, and Other Destinations. All of these functions have mostly georeferenced metadata repository. They are indexed alphabetically and can be filtered by category, making it more user-friendly to access individual articles. Attractions can be accessed on an offline GPS map along with the potential tourists' current locations so that they can easily find place of interest nearby just on a click [19]. The discussed websites or, 
mobile apps are enriched in multimedia based contents and impressive in terms of static online information. But in the era of Web 2.0 the potential tourists need interactive geoportals. The planning can be smooth for any potential tourist if they are able to search, contribute, share experience on top of an interactive georeferenced map.

\section{MYANMAR GEOSPATIAL INFORMATION SYSTEM FOR TOURISM (MGIST)}

According to Singh et al. (2011), GIS and tourism share a common characteristic, both cross the boundaries and areas of application. Geospatial Mashup has significant impact tourism information [16].

\section{A. System Architecture}

The proposed, "Myanmar Geospatial Information System for Tourism" (MGIST) is a client-side Mashup application following typical three-tier (view layer, logical layer and physical layer) architecture. The system has customized three levels of users. Each level of users has different levels of data accessibility and customization functions. Figure 8 (below) illustrates the different levels of users with their respective functionalities.

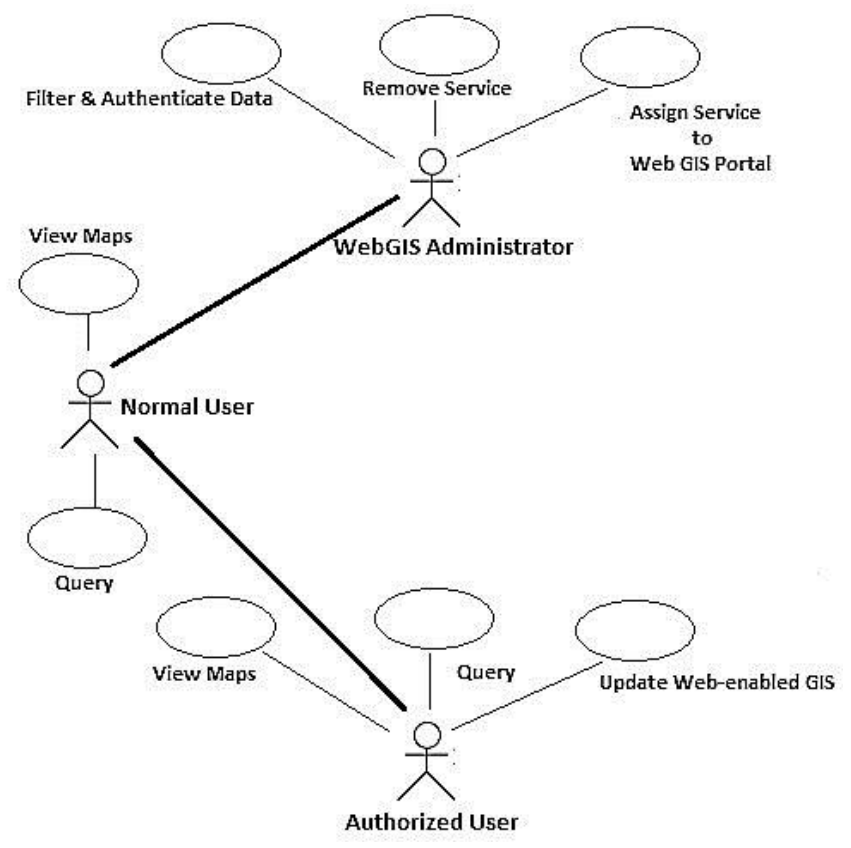

Fig. 8 Level of users in the proposed model

The primary focus in the process of Georeferenced Mashup development is on the user friendliness and the on-click availability of diverse global geo information in a common base map. So that anyone can share their local geospatial information in an online interactive common platform.

Open Geospatial Consortium (OGC) web service provides a vendor-independent interactive framework for web-based capturing, storing, and editing, analyzing and projecting geospatial data. Web Feature Service (WFS) and Web Map Service (WMS) are the two most important web service standards proposed by Open Geospatial Consortium, 2002. Figure 9 (below) depicts the workflow sequence for the proposed MGIST. It follows OGC service.

A series of "decision making ratings" will be used as the assessment criteria to study the characteristics of the destination and its potentiality to be a popular tourism site. The authorized users' web upload criteria and indicators will be revised suitably, so that the information sought may be cross checked in the administrator level. This series of "decision making ratings" will allow studying the relationship between the characteristics of the destination and its potentiality to be a popular tourism destination.

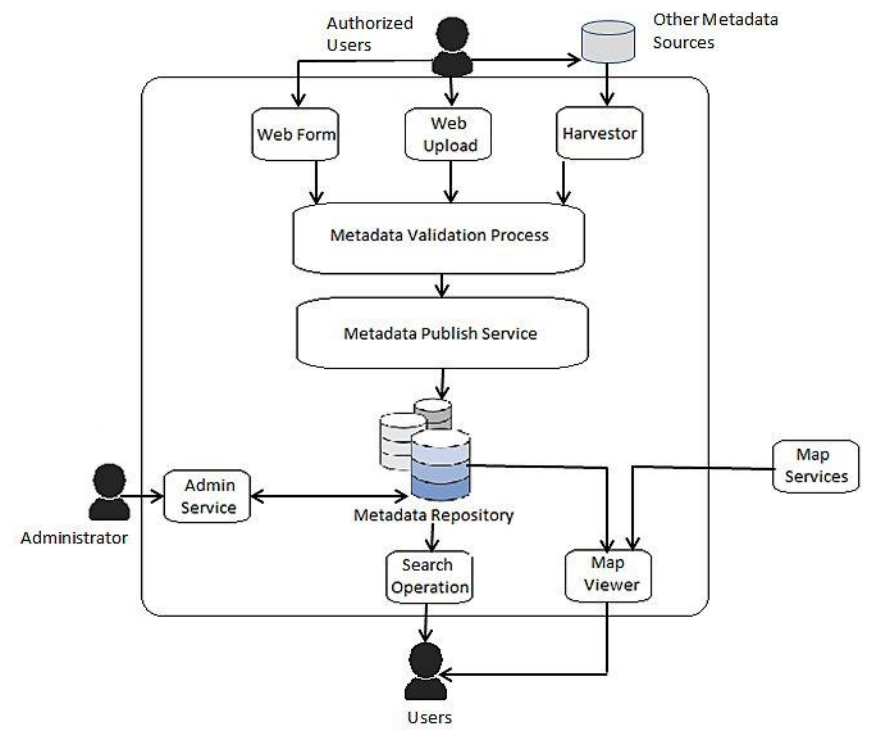

Source: Adapted from [20]

Fig. 9 Schematic Model of MGIST

\section{B. Interface Design}

The proposed model will have the following features:

- Basic home page

$\checkmark$ Filter by regions

- Display Mashup maps with user-controlled selected features of each location (Landmarks, Historical Sites, Beach, Food Places, and Hotels etc.). Multimedia based information will pop up on click.

- Filter places based on user preference

- Find direction and shortest distance between two locations.

- Information about the place (Aggregation of the results will be displayed to others)

$\checkmark$ Give ratings to places

$\checkmark$ Comment your experience

- Recommend or, create new and undiscovered potential tourist locations

- Update information about a location

- Administrator dashboard.

- Feedbacks and other inquiries- A help desk to improve the system. 


\section{System Components}

MGIST consists of five different component programs:

- QGIS as OGC Data Server (Open Source)

Compatible with Apache web server and having Python plugin support.

- PostGIS 2.0 Database spatial enable support

Dynamically updating layers in QGIS with PostGIS views. Spatial Database Management using PostgreSQL and PostGIS.

- MGIST application designed using PHP

Scripts (Hypertext Pre-processor) and Ajax. The user interface consists of a series of ".php" files which are rendered to the browser using Hypertext Mark-up Language (HTML). The Web application uses client-side scripts, i.e. it uses JQuery and JavaScript's files to execute instructions on the Web page.

Figure 10 (below) depicts the interaction of Database (PostgreSQL and PostGIS) with the Map and Web Server. But the loading is possible only when the Database tables are georeferenced.

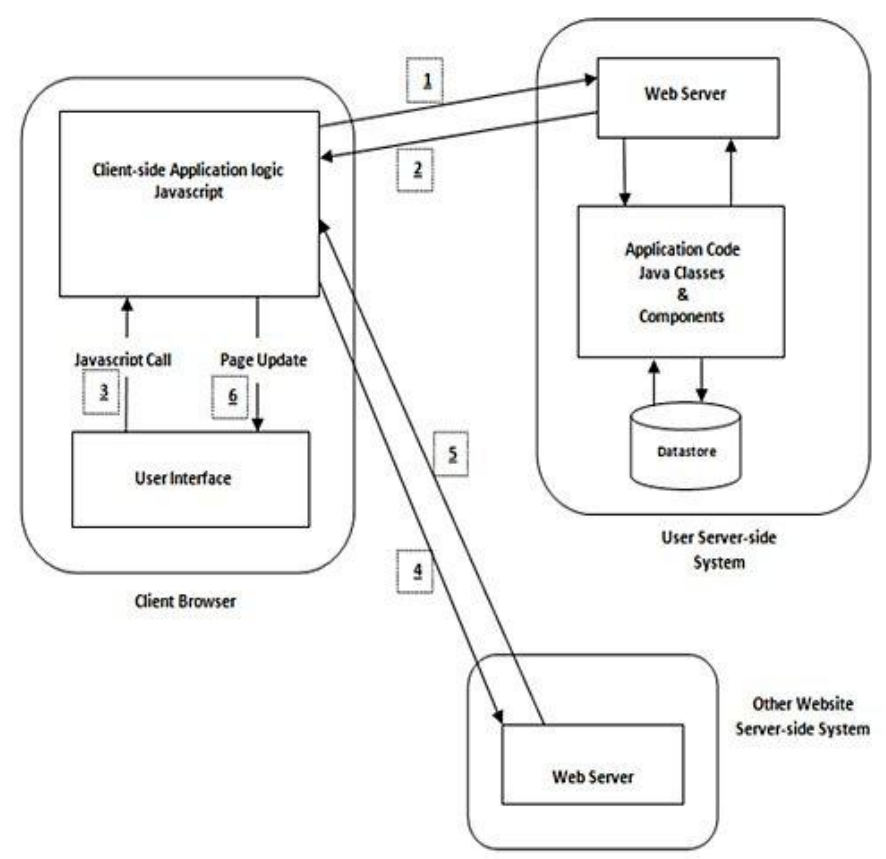

Source: Adapted from java.sun.com

Fig. 10 Client-side Mashup Architecture

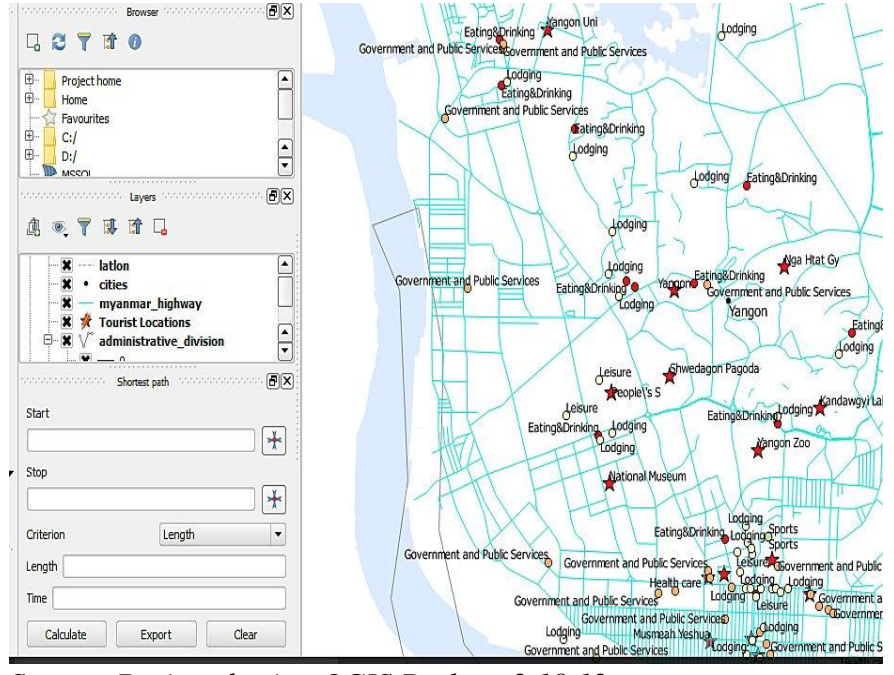

Source: Designed using QGIS Desktop 2.18.12

Fig. 11 Mashup Map of Yangon (Server Side)

Figure 11 demonstrates the server-side merging of a widerange georeferenced layers in the core QGIS platform. The example contains different tourism data layers for Yangon region of Myanmar.

The layers used in the above QGIS map are categorized as in figure 12 (below).

\begin{tabular}{lll} 
Symbol & $\Gamma$ & Legend \\
\hline $\mathbf{x}$ & 0 & Automotive \\
$\mathbf{x}$ & 0 & Eating\&Drinking \\
$\mathbf{x}$ & 0 & Government and Public Services \\
$\mathbf{x}$ & 0 & Health care \\
$\mathbf{x}$ & 0 & Leisure \\
$\mathbf{x}$ & 0 & Lodging \\
$\mathbf{x}$ & 0 & Night Life and Business \\
$\mathbf{x}$ & 0 & Sports \\
$\mathbf{x}$ & 0 & Tourism
\end{tabular}

Source: Designed using QGIS Desktop 2.18.12

Fig. 12 Attributes in Tourism Site Layer

After processing of the above (as shown in figure 11 QGIS) QGIS raw dataset it can be converted as a Keyhole Mark-up Language (KML) file and can be accessed using Google Earth Pro (another Open Source GIS software). Figure 13 (below) illustrates the KML output of the QGIS file. In this interface the user will have a wide range of options to filter out required information. In this example, railroads, parks, place of worship, restaurants, hotels, café, and tourist attractions are opted by the user. The geospatial Mashup interface depicts heterogeneous data set in the common platform with wide options of filtering the display parameters. Multimedia based information like the photo with important information and rating value about the location can also be accessed on click. When user selects a marker, the name of the place and a brief description of the place will be displayed. If user wants more information about the place just click to "See More" and they will be redirected to the information page might be updated by some other user. 


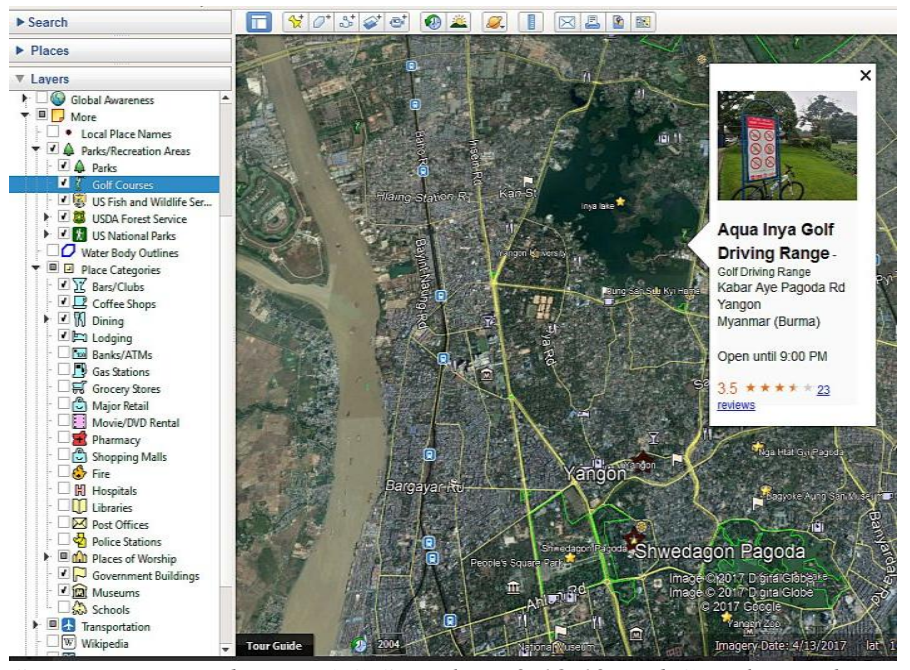

Source: Designed using QGIS Desktop 2.18.12 and Google Earth

Fig. 13 Mashup Map of Yangon (Client Side)

\section{CONCLUSION AND Discussion}

The available Myanmar tourism related information are static and solely dependent on the maintenance and update by the proprietors of the individual websites. The proposed "Myanmar Geospatial Information System for Tourism" (MGIST) will be "Information by the People for the People" and can be easily uploaded on web to respond to the potential tourist's query. It combines two important approaches. MGIST offers the common platform to publish proprietary data and use related metadata. It also helps in combining heterogeneous data from different sources and offers easy integration and reuse of user derived information sources like: Google Earth or, Open Street Map. The greatest advantage in this Participatory Mashups is that, the globally accessible Geo -Mashups contain the most recent information about the location. Even the tourists while travelling can update the Mashup information over the internet. This will be beneficial for other potential tourists. Data update and maintenance will not be the sole responsibility of any single organization. People will be able to share multimedia based information in this georeferenced platform, authenticated by some administrator. Thus, helping any potential tourist from any part of the globe to access most up to date information in a user-friendly geospatial information system. But the amenities and infrastructure facilities of the study areas are dynamic in nature, these information require edit, insert or delete operations whenever there is any update. The local community is the best contributor for this information set. The locals should be made aware of the online interactive geospatial information system and the functions available to update or add multimedia based resources for their local tourist locations. Awareness program will depict how this online geospatial information system can be strategically used as a promotional tool for the promotion and marketing of the potential tourist destinations.

The implementation of the proposed MGIST will provide unlimited and up to date information to everybody having access to internet helping them in taking better decisions to plan their trip. The system can be synonymous to "Information by the People for the People".

\section{REFERENCES}

[1] Travel \& Tourism Economic Impact 2017 Myanmar (2017). World Travel \& Tourism Council (WTTC). Retrieved from www.wttc.org//media/files/reports

[2] S. Chaudhuri, and P. Yamin, "Development of Tourism in Myanmar", Tourism Marketing: A Strategic Approach, CRC Press, USA, 2017

[3] Myanmar Tourism Statistics 2015. Ministry of Hotels and Tourism, Myanmar. Retrieved from www.myanmartourism.org

[4] Myanmar Tourism Master Plan 2013-2020. Final Draft Report June 2013. Ministry of Hotels and Tourism, Myanmar. Retrieved from www.myanmartourism.org/images/handbooks

[5] D. Buhalis, "Strategic use of information technologies in the tourism industry," in Tourism Management, 19th ed. vol. 5, 1998, pp. 409-421.

[6] P. Fu and J. Sun, Web GIS, Principles and Applications, ESRI Press, 2011, pp. $89-114$

[7] OGC, 2002, Web Feature Service Implementation Specification, version 1.0.0, OGC document 02-058

[8] S. Chaudhuri, and N. Ray, "Application of Web-Based Geographical Information System (GIS) In Tourism Development," Encyclopedia of Information Science and Technology, 4, IGI-Global, USA, 2015 https://doi.org/10.4018/978-1-4666-5888-2.ch692

[9] S. Chaudhuri, and N. Ray, "Application of Web-Based Geographical Information System (GIS) In Tourism Development", Encyclopedia of Information Science and Technology,3, IGI- Global, USA, 2014

[10] Mcadam D., "The Value and Scope of Geographical Information Systems in Tourism Management", Journal of Sustainable Tourism, 7(1), 1999, pp. 7792.

[11] Turk T., and Gumusay M. U., "GIS Design and Application for Tourism", International Archives of Photogrammetry Remote Sensing and Spatial Information Sciences, 35(4), 2004, pp. $485-488$.

[12] C. T. Alonso, S. Fern'andez, D. Berrueta, L.Polo, M. J. Fern'andez, and V. Morlan, "eZaragoza, A Tourist Promotional Mashup," Conf. AI Mashup Challenge 2010, co-located with the 7th Extended Semantic Web Conference, ESWC, Heraklion, Greece, 2010

[13] Anderson M. D., and Souleyrette R. R., "Pseudo dynamic travel model application to assess traveler Information", Transportation, 29, 2002, pp. 307-319. https://doi.org/10.1023/A:1015614208374

[14] Feick R. D., and Hall G. B., "The Application of a Spatial Decision Support System to Tourism Based Land Management in Small Island States", Journal of Travel Research, 39(2), 2000, pp. 163-171 https://doi.org/10.1177/004728750003900206.

[15] Berger H., Dittenbach M., Merkl D., Bogdanovych A., Simoff S., and Sierra C., "Opening new dimensions for e-Tourism", Virtual Reality, 11, 2007, pp. 75-87. https://doi.org/10.1007/s10055-006-0057-z

[16] S. P. Singh, J. Sharma, and P. Singh, "A Geo-Referenced Information System for Tourism (GeoRIST), International Journal of Geomatics and Geosciences, 2 (2), 2011, pp. 456-464

[17] Ministry of Hotels and Tourism, the Republic of the Union of Myanmar, Retrieved from tourism.gov.mm

[18] Myanmar Information Management Unit (MIMU), the Republic of the Union of Myanmar, Retrieved from themimu.info

[19] Burma (Myanmar) Free guide, Mobile Reference, Retrieved from play.google.com/store/apps/

[20] M.F. Goodchild, P. Fu, and P. Rich, "Sharing Geographic Information: An Assessment of the Geospatial One-Stop", Annals of the Association of American Geographers, 97(2), Blackwell Publishing, 2006, pp. 250-266.

[21] World Tourism Barometer 2017, World Tourism Organization (UNWTO), Retrieved from publications.unwto.org 